\title{
DE-STRESS: A user-friendly web application for the evaluation of protein designs
}

\author{
Michael J. Stam ${ }^{1}$ and Christopher W. Wood ${ }^{2 *}$. \\ 1. School of Informatics, University of Edinburgh, 10 Crichton St, Newington, \\ Edinburgh EH8 9AB. \\ 2. School of Biological Sciences, University of Edinburgh, Roger Land Building, \\ Edinburgh EH9 3FF. \\ * To whom correspondence should be addressed.
}

\section{Abstract}

De novo protein design is a rapidly growing field and there are now many interesting and useful examples of designed proteins in the literature. However, most designs could be classed as failures when characterised in the lab, usually as a result of low expression, misfolding, aggregation or lack of function. This high attrition rate makes protein design unreliable and costly. It is possible that some of these failures could be caught earlier in the design process if it were quick and easy to generate information and a set of high-quality metrics regarding designs, which could be used to make reproducible and data-driven decisions about which designs to characterise experimentally.

We present DE-STRESS (DEsigned STRucture Evaluation ServiceS), a web application for evaluating structural models of designed and engineered proteins. DE-STRESS has been designed to be simple, intuitive to use and responsive. It provides a wealth of information regarding designs, as well as tools to help contextualise the results and formally describe the properties that a design requires to be fit for purpose.

\section{Availability}

DE-STRESS is available for non-commercial use, without registration, through the following website: https://pragmaticproteindesign.bio.ed.ac.uk/de-stress/. Source code for the application is available on GitHub: https://github.com/wells-wood-research/de-stress. The 
data used to generate reference sets is available through a GraphQL API, with the following

\section{URL: https://pragmaticproteindesign.bio.ed.ac.uk/big-structure/graphql.}

\section{Introduction}

There has been rapid development in the field of de novo protein design over recent years, with more groups producing increasingly ambitious designs with complex behaviour, often applied in cellular environments (Ben-Sasson et al., 2021; Glasgow et al., 2019; Harrington et al., 2021; Herud-Sikimić et al., 2021; Pirro et al., 2020; Sesterhenn et al., 2020; VanDrisse et al., 2021; Pan et al., 2020).

Despite the great promise of de novo protein design, it remains the domain of highly specialist research groups, as there are significant barriers blocking broader adoption as a methodology. One major challenge is that only a fraction of designs adopt stable, folded structures when expressed (Huang et al., 2016), and it can be difficult to identify these models using the metrics calculated during the design process alone (Radom et al., 2018). This is especially challenging for designs with complex requirements that are needed for targeted applications.

Here we present DE-STRESS (DEsigned STRucture Evaluation ServiceS), a user-friendly web application for evaluating structural models of designed and engineered proteins. We aim to provide the user with as much information as possible about their designs before they select sequences to characterise experimentally. 


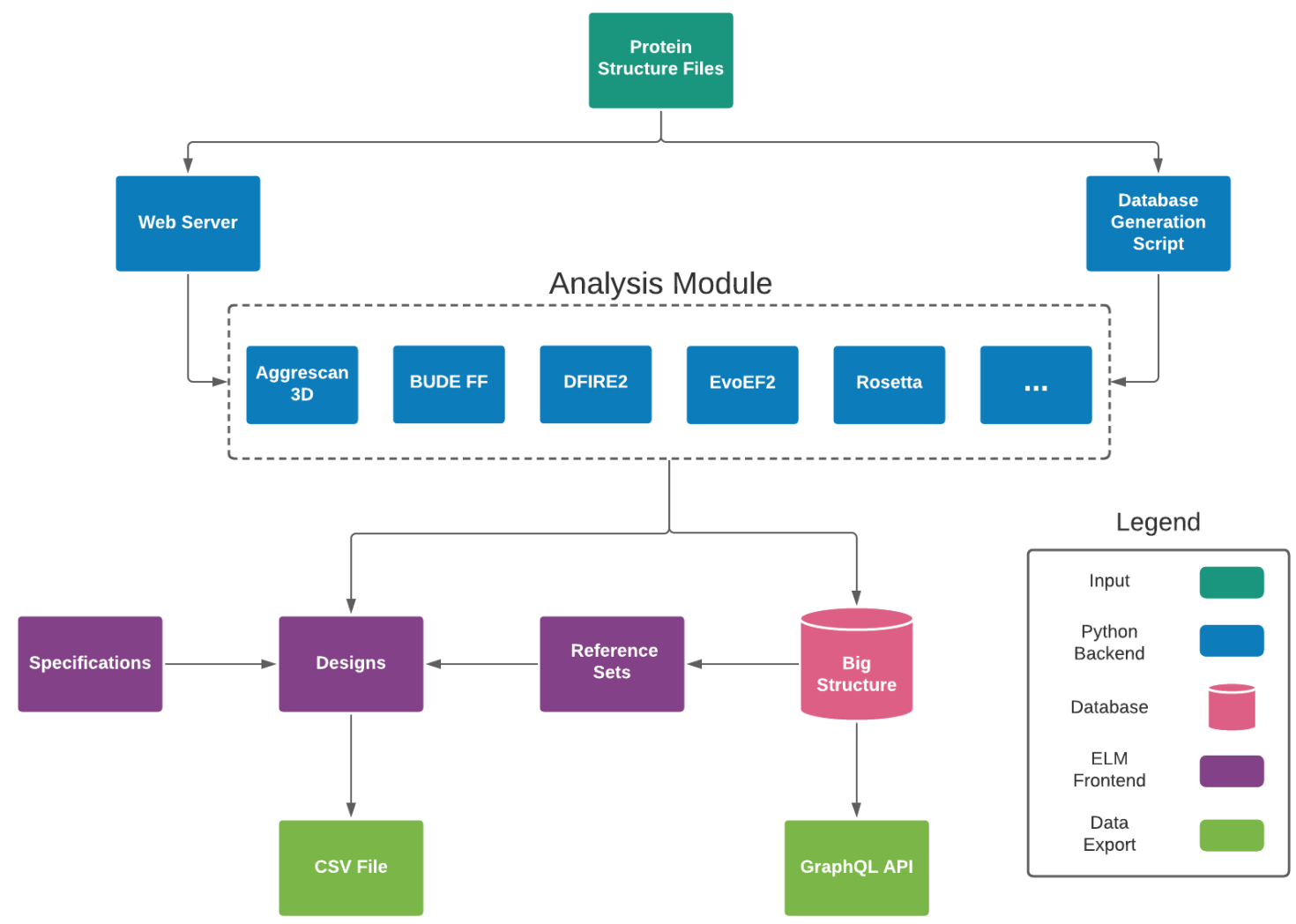

48

Figure 1: Overview of the DE-STRESS application architecture.

The DE-STRESS application consists of a simple and intuitive user interface, written in Elm/JavaScript, and a backend web stack, consisting of

Gunicorn/Flask/GraphQL/PostgreSQL (figure 1). The interface has three main sections that the user can explore: Designs, Reference Sets and Specifications.

On the Designs page (figure 2A), users can upload models of proteins (in PDB format) to the DE-STRESS server, where all the included metrics will be calculated for each design. Once the metrics have been calculated, an overview of the whole batch of designs is provided.

57 Detailed information can be viewed for each design (figure 2B), as well as a comparison to 58 the active Reference Set and Requirement Specification (vide infra). 


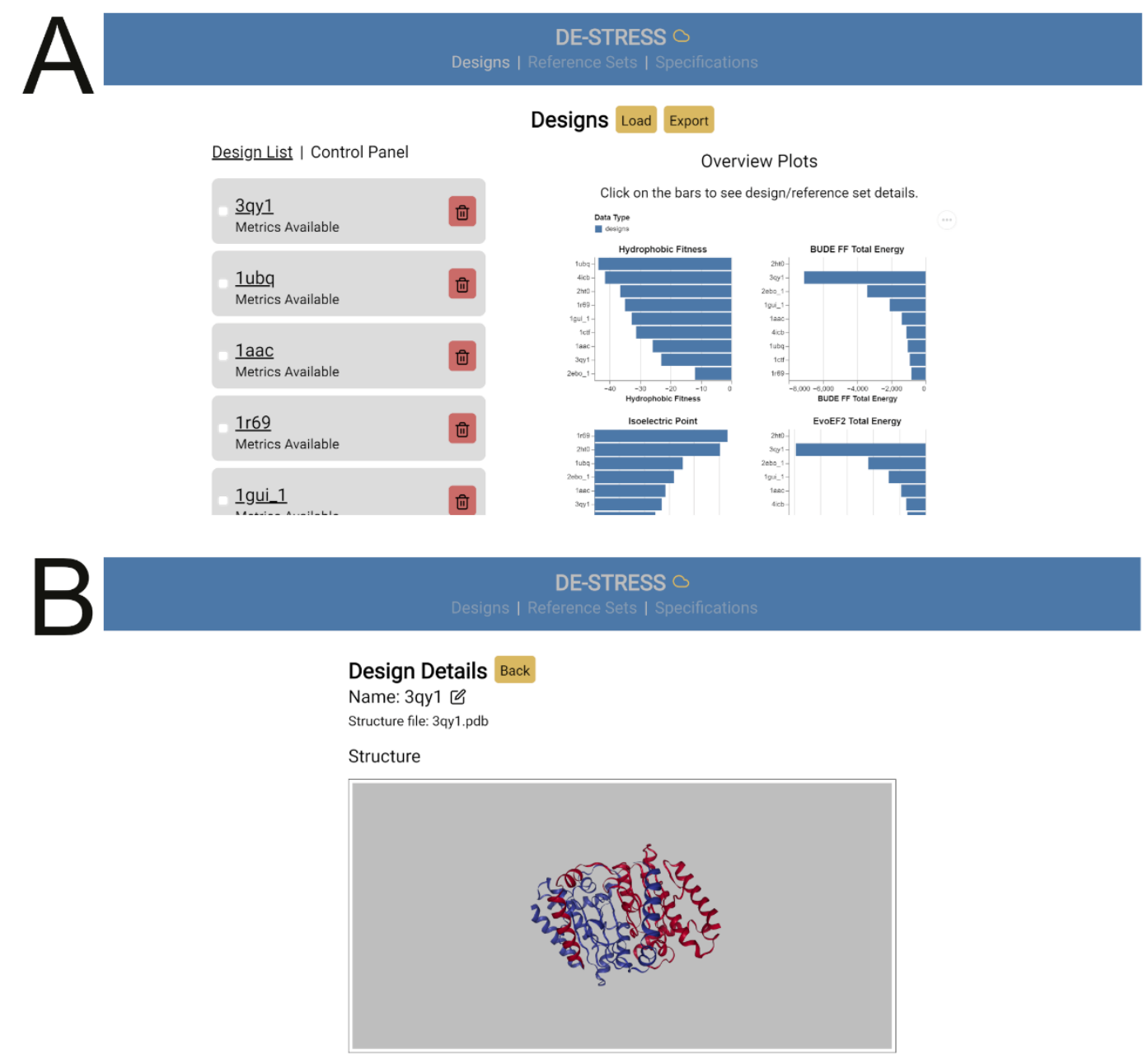

Figure 2: The DE-STRESS user interface. A) The "Designs" page allows users to upload designs, obtain information on the whole batch of designs and download a CSV file containing their results. B) Detailed information on specific designs is offered on the "Design Details" page.

On the Reference Sets page, users can define a set of known protein structures from the PDB (Berman et al., 2003), which can be used as a basis of comparison for their designs. We have precalculated the metrics included in DE-STRESS for the biological units, as defined by the PDBe (http://ftp.ebi.ac.uk/pub/databases/pdb/data/biounit/), of 82,010 protein structures. The remaining structures in the PDB either did not contain protein, contained formatting errors in the PDB file or, in the case of large structures, failed to return results within a reasonable timeframe. Similar restrictions have been placed on models that can be 
uploaded to the webserver, in order to ensure server stability. Using these data, the user can define their own reference sets by submitting a list of PDB accession codes, enabling them to compare their designs to relevant structures. Additionally, two default reference sets are provided as an example, based on high-quality structures from Top500 (Hobohm and Sander, 1994) and Pisces (Wang and Dunbrack, 2003). Finally, users can also define reference sets from models that they have uploaded to the server, allowing the creation of reference sets from unpublished structures.

Once a reference set has been defined, aggregated metrics are presented alongside the metrics for the user's designs. All the data used to generate the reference sets are available to search and download, programmatically and interactively, through a GraphQL API available at the following url: https://pragmaticproteindesign.bio.ed.ac.uk/big-

\section{structure/graphql.}

Reference sets are intended to provide context for the metrics returned by DE-STRESS, and while we have provided some example reference sets of high-quality crystal structures, manually defining a reference set is much more useful. For example, if you are designing coiled-coil proteins, comparing your designs to similar structures is likely to be more useful than a general set of proteins, as the metrics and other information, such as sequence composition, will be directly comparable. Furthermore, it is worth noting that designs with metrics that fall outside of the range observed for known protein structures may still express, fold and be functional. In certain instances, the designer may be actively selecting designs with metrics that fall outside of those observed for natural proteins, in fact this is very often the purpose of designing proteins de novo.

The Specifications page allows the user to define "Requirement Specifications", which encapsulate the properties their designs should have in order to be fit for purpose. The user can define complex rules, such as nested conditional properties, that can be used to filter designs, alongside associated metadata. We plan to expand the role of the specifications in 
the future, allowing the user to capture more information about their design intent and export the specification to be used by other programmes.

\begin{tabular}{|c|c|c|c|}
\hline Evaluation Method & Type & $\begin{array}{c}\text { Scoring } \\
\text { Convention }\end{array}$ & Reference \\
\hline Packing Density & \multirow[t]{2}{*}{ Geometric Analysis } & + +ve & (Weiss, 2007) \\
\hline Hydrophobic Fitness & & -ve & (Huang et al., 1995) \\
\hline BUDE FF & \multirow[t]{3}{*}{$\begin{array}{l}\text { All-Atom Scoring } \\
\text { Function }\end{array}$} & $-v e$ & $\begin{array}{l}\text { (Mclntosh-Smith et al., 2012, } \\
2015 \text { ) }\end{array}$ \\
\hline EvoEF2 & & -ve & (Huang et al., 2020, 2) \\
\hline Rosetta & & -ve & (Alford et al., 2017) \\
\hline DFIRE2 & Statistical Potential & -ve & (Yang and Zhou, 2008) \\
\hline Aggrescan3D & $\begin{array}{l}\text { Aggregation } \\
\text { Propensity }\end{array}$ & $-v e$ & (Kuriata et al., 2019) \\
\hline ISAMBARD & Basic Information & $N / A$ & (Wood et al., 2017) \\
\hline DSSP & $\begin{array}{l}\text { Secondary-Structure } \\
\text { Assignment }\end{array}$ & $N / A$ & $\begin{array}{l}\text { (Kabsch and Sander, 1983; } \\
\text { Touw et al., 2015) }\end{array}$ \\
\hline
\end{tabular}

Table 1: Model evaluation methods included in DE-STRESS. The scoring convention indicates whether the score is considered more favourable if it is lower (-ve) or higher (+ve).

A variety of external software packages are used by the DE-STRESS web server to calculate metrics for uploaded models (table 1, supplementary table 1), with detailed and upto-date information on the version and command used to run the software provided in the supplementary material and glossary page of the application. Basic information is extracted using ISAMBARD (Wood et al., 2017), such as the isoelectric point and composition of the sequence, as well as implementations of metrics from the literature, such as packing density (Weiss, 2007) and hydrophobic fitness (Huang et al., 1995). In addition to these metrics, DESTRESS applies a range of scoring functions that have either been developed specifically for protein design or have been applied to design proteins, such as BUDE FF (MclntoshSmith et al., 2012, 2015; Thomson et al., 2014), EvoEF2 (Huang et al., 2020b, 2, 2020a), Rosetta (Alford et al., 2017; Cao et al., 2020) and DFIRE2 (Yang and Zhou, 2008; Negron and Keating, 2014). Finally, we use Aggrescan3D (Kuriata et al., 2019) to calculate an aggregation propensity score for protein structures, as aggregation is a common failure 
mode for designed and engineered proteins (Marques et al., 2021). For detailed information regarding the metrics included see supplementary tables $2-11$. These metrics are presented on the Design Details page, alongside a visualisation of the model, using the NGL JavaScript library (Rose and Hildebrand, 2015; Rose et al., 2016), and other information such as secondary structure assignment using DSSP (Kabsch and Sander, 1983; Touw et al., 2015).

A privacy first approach has been taken when implementing DE-STRESS. No login is required to use the application and no data regarding the user, or their designs, are stored on our server. Designs are submitted directly to an in-memory job queue, with no associated metadata, and the results are returned directly to the user. All data regarding the user's designs are stored locally on the device used to access the website and can be exported to a CSV file for further analysis. With this architecture, we aim to give the user confidence in submitting their designs to the server. However, if they would like to take further steps to ensure that no one could access their data, they can run a local instance of the web application, which we have made as simple as possible by containerising the application.

We envisage that, for many users, DE-STRESS will be useful for generating descriptive information and statistics that could be manually examined to choose designs that meet the needs of their application. Beyond this, the datasets that DE-STRESS creates could be useful for automatic identification of high-quality models using data-driven methods. As a simple example of this, we attempted to discern experimentally-determined structures from decoys that are used to benchmark protein-folding algorithms. The dataset and the associated scripts for performing this analysis are available on GitHub:

\section{https://github.com/wells-wood-research/stam-m-wood-c-de-stress-2021.}

Using the DE-STRESS web application, we generated and exported metrics for a random sample of 9 experimentally-determined structures, along with 360 decoys (40 per structure) from a set that was previously generated by 3DRobot. 3DRobot produces structurally diverse and well-packed decoy structures that are difficult to discern from experimentally- 

radius of gyration, as well as more complex metrics such as statistical potentials (Deng et al., 2016). We also included alternative structures for each of these proteins (supplementary table 14), identified through the structural similarity search tool on the RSCB PDB, to ensure that the findings were robust to minor variations in the structure.
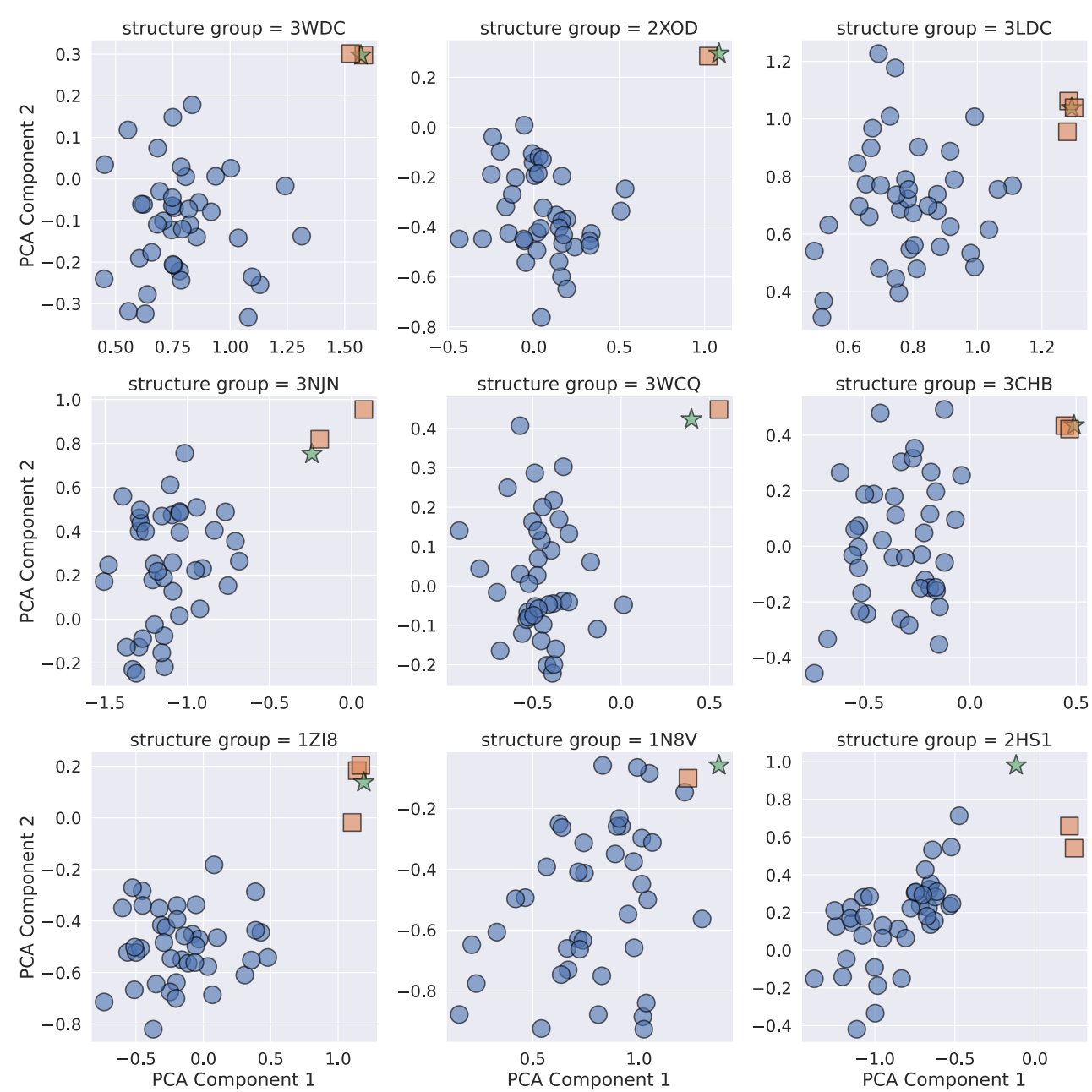

Figure 3: Principal component analysis of DE-STRESS metrics generated for experimentally-determined structures (stars), folding decoys (circles) and alternative crystallographic structures of the protein (squares).

Firstly, before performing PCA, various metrics were excluded from the data set. Categorical and discrete variables were excluded as PCA requires continuous variables, and metrics that were constant for a structure were excluded. This is because these metrics provided no information that could be used to distinguish between the decoys and experimentally- 
determined structures. In addition to this, as the energy values are dependent on the size of the protein structure, we normalised for length by dividing all the energy values by the number of residues in the structure. A full list of the metrics included in the analysis is shown in the-supplementary table 12. Before performing PCA, the metrics were normalised using min-max normalisation. The first two principal components, which explained $60 \%$ of the variance in the data, were plotted against each other to explore how well the DE-STRESS metrics differentiated the experimentally-determined structures from the decoys (figure 3). The additional crystallographic structures were also included in these plots.

From the plots in figure 3, we see that the experimentally-determined structures, shown as stars, are generally distinct from the decoys and usually have the largest values for both principal components 1 and 2. In addition to this, the additional crystallographic structures, shown as squares, are close to the experimentally-determined structures used to generate the 3DRobot set. This analysis suggests that the DE-STRESS metrics are capturing properties of the structural models that can be used to distinguish "native-like" structures from decoys.

To understand which metrics differentiated the experimentally-determined structures from the decoys, we examined the relative contribution of individual DE-STRESS metrics to the first two principal components (supplementary table 13). It was found that the major contributors to PC1 were the short range and long-range hydrogen bonding terms in from the Rosetta forcefield and the total BUDE forcefield energy value. The major contributors to PC2 were the average score of Aggrescan3D, the steric component of the BUDE forcefield and a solvation term from the Rosetta forcefield. Long-range hydrogen bonding was highlighted by the developers of 3DRobot as a key differentiating feature between native structures and decoys, and maintaining this property was a key to creating native-like decoys. However, while analysis performed in the 3DRobot publications shows that their decoys performed significantly better in this regard than previous decoy sets (Deng et al., 2016), our results indicate that there is still some detectable difference between the 
experimentally-determined structures and the decoys. The fact that Aggrescan3D is a key contributor to PC2 might indicate that the surface properties of the decoys are significantly different from the native structures, and this could indicate a potential route to improve the process used to create decoy models.

\section{Conclusions}

DE-STRESS enables both non-experts and seasoned protein designers to rapidly evaluate their designs, providing a framework for making reproducible, data-driven decisions about which design to take forward for experimental characterisation. While some protocols and applications have been developed to meet similar needs as DE-STRESS (Bernhofer et al., 2021; Guffy et al., 2018; Yallapragada et al., 2020, 2), none of them have the same breadth of metrics and tools, all packaged in a user-friendly web application. However, the metrics included in the initial version of DE-STRESS are certainly not exhaustive: general methods for assessing the quality of a protein model (Vriend, 1990; Wang et al., 2019; Williams et al., 2018; Laskowski et al., 1993), as well as more specialised methods for assessing design quality, such as analysis of covariation similarity with homologs (Ollikainen and Kortemme, 2013; Ó Conchúir et al., 2015; Ludwiczak et al., 2018), could be useful additions in future releases. We believe that our analysis of decoy structures demonstrates that the metrics included in this initial version of DE-STRESS are useful and can be used to identify highquality models, but further experimental work is required to determine whether this translates into a reduced failure rate of designs taken into the lab, which would meet our ultimate aim of increasing the efficiency of the protein-design process and making it more accessible and reliable as a technique.

\section{Acknowledgements}

The authors would like to thank Lynne Regan, Dek Woolfson for feedback on the manuscript/application and Kathryn Shelly for feedback on the application. We would also like to thank the UoE School of Biological Sciences IT department for infrastructure support, 
and the developers of the software used to generate many of the metrics included in DE-

STRESS.

\section{Funding}

CWW is supported by an Engineering and Physical Sciences Research Council Fellowship (EP/S003002/1). Michael Stam is supported by the United Kingdom Research and Innovation (grant EP/S02431X/1), UKRI Centre for Doctoral Training in Biomedical AI at the University of Edinburgh, School of Informatics. This work was supported by the Wellcome Trust-University of Edinburgh Institutional Strategic Support Fund (ISSF3).

\section{References}

Alford,R.F. et al. (2017) The Rosetta All-Atom Energy Function for Macromolecular Modeling and Design. J. Chem. Theory Comput., 13, 3031-3048.

Ben-Sasson,A.J. et al. (2021) Design of biologically active binary protein 2D materials. Nature, 589, 468-473.

Berman,H. et al. (2003) Announcing the worldwide Protein Data Bank. Nature Structural \& Molecular Biology, 10, 980-980.

Cao,L. et al. (2020) De novo design of picomolar SARS-CoV-2 miniprotein inhibitors. Science, 370, 426-431.

Deng,H. et al. (2016) 3DRobot: automated generation of diverse and well-packed protein structure decoys. Bioinformatics, 32, 378-387.

Glasgow,A.A. et al. (2019) Computational design of a modular protein sense-response system. Science, 366, 1024-1028.

Harrington,L. et al. (2021) De novo design of a reversible phosphorylation-dependent switch for membrane targeting. Nature Communications, 12, 1472.

Herud-Sikimić,O. et al. (2021) A biosensor for the direct visualization of auxin. Nature, 1-5.

Hobohm,U. and Sander,C. (1994) Enlarged representative set of protein structures. Protein Science, 3, 522-524.

Huang,E.S. et al. (1995) Recognizing native folds by the arrangement of hydrophobic and polar residues. J Mol Biol, 252, 709-720.

Huang,P.-S. et al. (2016) The coming of age of de novo protein design. Nature, 537, 320327.

Huang,X. et al. (2020a) De novo design of protein peptides to block association of the SARS-CoV-2 spike protein with human ACE2. Aging (Albany NY), 12, 11263-11276.

Huang,X. et al. (2020b) EvoEF2: accurate and fast energy function for computational protein design. Bioinformatics, 36, 1135-1142.

Kabsch,W. and Sander,C. (1983) Dictionary of protein secondary structure: Pattern recognition of hydrogen-bonded and geometrical features. Biopolymers, 22, 25772637.

Kuriata,A. et al. (2019) Aggrescan3D standalone package for structure-based prediction of protein aggregation properties. Bioinformatics, 35, 3834-3835.

Laskowski,R.A. et al. (1993) PROCHECK: a program to check the stereochemical quality of protein structures. Journal of Applied Crystallography, 26, 283-291. 
bioRxiv preprint doi: https://doi.org/10.1101/2021.04.28.441790; this version posted November 4, 2021. The copyright holder for this preprint (which was not certified by peer review) is the author/funder, who has granted bioRxiv a license to display the preprint in perpetuity. It is made available under aCC-BY 4.0 International license.

Ludwiczak,J. et al. (2018) Combining Rosetta with molecular dynamics (MD): A benchmark of the MD-based ensemble protein design. Journal of Structural Biology, 203, 54-61.

Marques,S.M. et al. (2021) Web-based tools for computational enzyme design. Current Opinion in Structural Biology, 69, 19-34.

McIntosh-Smith,S. et al. (2012) Benchmarking Energy Efficiency, Power Costs and Carbon Emissions on Heterogeneous Systems. The Computer Journal, 55, 192-205.

Mclntosh-Smith,S. et al. (2015) High performance in silico virtual drug screening on manycore processors. The International Journal of High Performance Computing Applications, 29, 119-134.

Negron,C. and Keating,A.E. (2014) A Set of Computationally Designed Orthogonal Antiparallel Homodimers that Expands the Synthetic Coiled-Coil Toolkit. J. Am. Chem. Soc., 136, 16544-16556.

Ó Conchúir,S. et al. (2015) A Web Resource for Standardized Benchmark Datasets, Metrics, and Rosetta Protocols for Macromolecular Modeling and Design. PLoS One, 10, e0130433.

Ollikainen,N. and Kortemme,T. (2013) Computational Protein Design Quantifies Structural Constraints on Amino Acid Covariation. PLOS Computational Biology, 9, e1003313.

Pan,X. et al. (2020) Expanding the space of protein geometries by computational design of de novo fold families. Science, 369, 1132-1136.

Pirro,F. et al. (2020) Allosteric cooperation in a de novo-designed two-domain protein. PNAS, 117, 33246-33253.

Radom,F. et al. (2018) Assessment of ab initio models of protein complexes by molecular dynamics. PLOS Computational Biology, 14, e1006182.

Rose,A.S. et al. (2016) Web-based molecular graphics for large complexes. In, Proceedings of the 21st International Conference on Web3D Technology, Web3D '16. Association for Computing Machinery, New York, NY, USA, pp. 185-186.

Rose,A.S. and Hildebrand,P.W. (2015) NGL Viewer: a web application for molecular visualization. Nucleic Acids Research, 43, W576-W579.

Sesterhenn,F. et al. (2020) De novo protein design enables the precise induction of RSVneutralizing antibodies. Science, 368.

Thomson,A.R. et al. (2014) Computational design of water-soluble $\alpha$-helical barrels. Science, 346, 485-488.

Touw,W.G. et al. (2015) A series of PDB-related databanks for everyday needs. Nucleic Acids Research, 43, D364-D368.

VanDrisse,C.M. et al. (2021) Computationally designed pyocyanin demethylase acts synergistically with tobramycin to kill recalcitrant Pseudomonas aeruginosa biofilms. PNAS, 118.

Vriend,G. (1990) WHAT IF: a molecular modeling and drug design program. J Mol Graph, 8, 52-56, 29.

Wang,G. and Dunbrack,R.L.,Jr (2003) PISCES: a protein sequence culling server. Bioinformatics, 19, 1589-1591.

Wang,W. et al. (2019) PSICA: a fast and accurate web service for protein model quality analysis. Nucleic Acids Research, 47, W443-W450.

Weiss,M.S. (2007) On the interrelationship between atomic displacement parameters (ADPs) and coordinates in protein structures. Acta Crystallogr D Biol Crystallogr, 63, 1235-1242.

Williams,C.J. et al. (2018) MolProbity: More and better reference data for improved all-atom structure validation. Protein Sci, 27, 293-315.

Wood,C.W. et al. (2017) ISAMBARD: an open-source computational environment for biomolecular analysis, modelling and design. Bioinformatics, 33, 3043-3050.

Yang,Y. and Zhou,Y. (2008) Ab initio folding of terminal segments with secondary structures reveals the fine difference between two closely related all-atom statistical energy functions. Protein Science, 17, 1212-1219. 